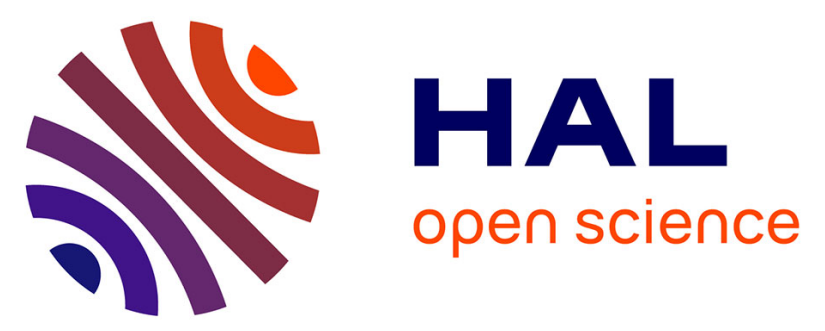

\title{
Regards croisés sur l'évaluation économique du patrimoine naturel: de la ressource d'autorité à la petite fabrique des valeurs?
}

Vincent Banos, Bénédicte Rulleau

\section{- To cite this version:}

Vincent Banos, Bénédicte Rulleau. Regards croisés sur l'évaluation économique du patrimoine naturel: de la ressource d'autorité à la petite fabrique des valeurs?. Annales de géographie, 2014, 699, pp.11931214. hal-01690506

\author{
HAL Id: hal-01690506 \\ https://hal.science/hal-01690506
}

Submitted on 23 Jan 2018

HAL is a multi-disciplinary open access archive for the deposit and dissemination of scientific research documents, whether they are published or not. The documents may come from teaching and research institutions in France or abroad, or from public or private research centers.
L'archive ouverte pluridisciplinaire HAL, est destinée au dépôt et à la diffusion de documents scientifiques de niveau recherche, publiés ou non, émanant des établissements d'enseignement et de recherche français ou étrangers, des laboratoires publics ou privés. 


\title{
Regards croisés sur l'évaluation économique du patrimoine naturel : de la ressource d'autorité à la petite fabrique des valeurs ${ }^{1}$ ?
}

\author{
On the patrimonialisation of the environment and of its \\ economic values : two shared perspectives
}

\author{
Vincent Banos \\ Ingénieur de recherche en géographie, Irstea - Unité ADBX

\section{Bénédicte Rulleau} \\ Maître de conférences en sciences économiques - Laboratoire REEDS - Université de Versailles \\ Saint-Quentin-en-Yvelines
}

Résumé Identifié et circonscrit depuis le $\mathrm{XIX}^{\mathrm{e}}$ siècle aux monuments historiques et aux curiosités naturelles remarquables, le patrimoine n'a cessé de s'étendre jusqu'à enserrer aujourd'hui des paysages et des écosystèmes. Mais parallèlement, un autre changement majeur s'opère, à savoir l'intégration du patrimoine, d'abord reconnu pour ses vertus symboliques, à la sphère des échanges marchands. Or, si ce registre de justification de la conservation patrimoniale a pu être étudié dans le cadre du développement territorial, peu de travaux ont à ce jour porté sur les implications sociales et politiques d'une économisation du patrimoine naturel. En procédant à une revue de la littérature et en s'inspirant de diverses études de cas, l'objectif de ce regard croisé entre un géographe et une économiste est de proposer une réflexion sur les usages sociaux de cette monétarisation de l'environnement et sa participation à la "petite fabrique » du patrimoine naturel. Nous verrons ainsi comment l'évaluation économique, bien que vivement critiquée, est progressivement devenue un cadre normatif des politiques publiques et un opérateur stratégique des processus de patrimonialisation. Loin de rester circonscrit aux seuls pouvoirs publics, cet outil est désormais instrumentalisé, par une diversité d'acteurs, pour imposer, négocier ou co-construire des valeurs.

Abstract Identified since the $19^{\text {th }}$ Century, the concept of heritage is constantly expanding. Everything is now likely to become patrimonial, even the environment. However, at the same time, this heritage is now integrated into the sphere of commercial exchange whereas it was initially recognized for its symbolic qualities. This new register of justification has been studied in the case of territorial development. However, to the best of our knowledge, little work has concerned the social and political implications of the economic valuation of the natural heritage. This paper proposes the shared perspectives of a geographer and an economist. Based on a literature review and various case studies, it aims at discussing the social uses of economic valuation. It also questions how this tool takes part in the creation of the economic values of the natural heritage. We analyse how the monetary valuation, although highly criticized, gradually became a normative framework

1 Les auteurs tiennent à remercier les organisateurs et les participants du colloque "Patrimonialiser la nature : valeurs et processus » qui s'est tenu à Pau du 7 au 9 septembre 2011, ainsi que les relecteurs pour leurs précieux conseils. 
for designing public policies and an additional resource of authority in patrimonial expansion. But the transformation in public decision making encourages taking a better look at the way this tool is now mobilized in order to enforce, negotiate or co-build new values.

Mots-clés patrimonialisation, nature, évaluation économique, monétarisation de l'environnement, usages, valeurs, échelles, légitimité.

Keywords natural heritage, environment, monetary valuation, social uses, values, scales, legitimacy.

\section{Introduction}

Identifié et circonscrit depuis le $\mathrm{XIX}^{\mathrm{e}}$ siècle aux monuments historiques et aux curiosités naturelles remarquables, collections d'artefacts exceptionnels et irremplaçables, le patrimoine n'a cessé de s'étendre, des traces les plus humbles de la vie quotidienne jusqu'à la biodiversité. Pour Heinich (2009), l'élargissement de la notion de patrimoine serait donc tout à la fois catégoriel (des "cathédrales » de la nature à la biodiversité), chronologique (du passé aux générations futures), topographique (des sites aux écosystèmes) et conceptuel (de l'unique au typique d'un ensemble). Mais, cette spectaculaire extension s'accompagne également d'une évolution des référentiels et registres de justification de l'ordre patrimonial avec un effacement de certains de ses moteurs originels, tels que les identités nationales ou «le naturalisme sensible » (Depraz, 2008), et, inversement, une emprise croissante des argumentaires économiques. Loin d'être nouvelles, ces logiques de valorisation se sont cependant généralisées en réussissant notamment à renouveler l'idée selon laquelle le patrimoine se reconnaîtrait au fait que sa conservation suppose des sacrifices (Babelon et Chastel, 1981). En effet, l'économie a su proposer et formater des «solutions » pour réconcilier conservation et usages en pensant le patrimoine comme un ensemble de biens qui, tout en générant des dépenses et des manques à gagner, peut être aussi une ressource profitant aux individus et au développement territorial. Le recours désormais quasi systématique aux «qualités » patrimoniales dans la construction et la mise en désir des territoires démontre tout le succès actuel de cette grille de lecture. Dans un contexte de concurrence généralisée entre territoires, ses capacités supposées à conférer des qualités spécifiques aux objets et produits érigent ainsi le patrimoine en facteur d'attractivité et de compétitivité essentiel (Landel et Sénil, 2009). Bien que pouvant remettre en question la conception d'un patrimoine qui devrait «se trouver exclu du circuit des valeurs marchandes pour sauver sa propre valeur symbolique »(Jeudy, 2001), ces approches suscitent un certain enthousiasme lorsqu'elles s'inscrivent dans le cadre de la co-dialectique du territoire et du patrimoine (Pecqueur, 2002 ; Barrère et al., 2005 ; Landel et Senil, 2009). Mais il en va tout autrement d'un autre vecteur majeur de l'économisation du patrimoine, l'évaluation économique de l'environnement : bien qu'occupant une place importante dans les textes réglementaires et dans l'argumentaire des grandes 
organisations environnementales, voire des gestionnaires, ses usages restent très controversés et paradoxalement rarement questionnés.

Émergeant aux États-Unis au début du $\mathrm{XX}^{\mathrm{e}}$ siècle, les méthodes consistant à doter les services naturels non marchands d'une valeur ayant la propriété d'un prix se sont progressivement déployées en raison d'une rationalisation croissante des modalités de l'action publique, mais aussi d'un certain constat d'échec des politiques de gestion des ressources naturelles et d'une sensibilisation croissante du monde conversationniste au discours néo-libéral (Millian et Rodary, 2010). Pour ses défenseurs, cette approche constituerait une alternative efficace permettant non seulement de rendre compte des services et du bien-être générés par le patrimoine naturel, mais aussi d'éviter sa surexploitation en révélant le coût de sa dégradation (Desaigues et Point, 1993). Cette posture suscite d'importantes discussions et de vives critiques, le plus souvent d'ailleurs au sein même du champ économique (Harribey, 1997 ; Godard, 2004 ; Bürgenmeier, 2008 ; Vivien, 2009). La tentation de calculer les différentes valeurs du patrimoine à partir d'axiomes théoriques qui, issus de la pensée néoclassique, mettent l'accent sur des individus rationnels et leurs capacités à maximiser la satisfaction que leur procure un bien (Homo xconomicus), cristallise bien des reproches. De fait, il serait théoriquement possible de donner un prix à tout, qu'il s'agisse de la survie d'une espèce ou d'une portion d'autoroute, pour au final les considérer comme comparables (Milanesi, 2010). Au-delà des difficultés méthodologiques posées par l'hétérogénéité des valeurs du patrimoine et de ses acteurs, l'économie de l'environnement se trouve surtout accusée de promouvoir une conception anthropocentrique et instrumentale de la nature (Harribey, 1997) et d'incarner l'emprise croissante de la rationalité marchande dans tous les domaines de l'existence humaine, y compris ses aspects politiques (Dardot et Laval, 2009).

Ces critiques constituent indéniablement des limites importantes aux démarches de monétarisation du patrimoine naturel mais, en se plaçant sur un plan moral et éthique, elles induisent deux biais potentiels. Premièrement, le rejet de l'existence même de ces méthodes laisse souvent dans l'ombre l'usage social qui en est fait et ne permet pas de considérer avec sérieux les effets qu'elles produisent (Callon et Latour, 2013). Ensuite, les critiques laissent parfois à penser que, par opposition aux logiques utilitaristes, la patrimonialisation serait viscéralement vertueuse. Or, c'est bien parce que le patrimoine possède cette faculté caractéristique à se faire oublier comme construction sociale pour s'imposer comme une évidence indiscutable et consensuelle (Di Méo, 1995) qu'il est important d'insister sur les logiques sociales sous-jacentes. Les géographes notamment se sont attachés à ce travail de déconstruction critique en montrant comment, derrière les rhétoriques holistiques du bien commun, la patrimonialisation était toujours une sélection, un pouvoir d'énoncer et de légitimer une certaine conception de l'espace et de l'être ensemble (Veschambre, 2007 ; Banos, 2008). Bien que de plus en plus ouvert à une diversité d'acteurs, d'échelles et d'objets d'intervention, ce processus narratif reste une idéologie 
avec ses rapports de force et ses inégalités, ses mises en scènes et ses opérateurs (Banos, 2010).

Dans le sillage de ces analyses critiques, l'objectif de cet article est donc d'interroger l'évaluation du patrimoine naturel dans ses rôles d'opérateur stratégique et de registre de légitimation des processus de patrimonialisation. Ainsi, nous nous intéressons à l'usage social de cette démarche normative, en ce qu'elle est proclamée utile, voire indispensable, aux procédures de choix publics. Comment les acteurs s'en saisissent-ils ? Est-elle seulement une ressource d'autorité supplémentaire accentuant l'emprise des représentations dominantes ou permet-elle à certains de se faire entendre et de réinsérer de la pluralité dans l'ordre patrimonial ? Si ce questionnement pragmatique n'est pas nouveau (Godard, 1990), peu de travaux (Colon et al., 2009 ; Laurans et al., 2013a) ont tenté de décrire les usages sociaux de cette nouvelle forme de légitimation du patrimoine naturel, avec ses contraintes et ses ressources. Sur la base d'une revue de la littérature en économie, géographie et aménagement et la recension d'études de cas, nous visons à contribuer modestement à ce défi et à décaler le regard sur l'expansion patrimoniale, entre valeurs économiques, singularités géographiques et pluralités sociales. Nous nous intéresserons premièrement à la construction de l'évaluation en cadre normatif, de sa place croissante dans les politiques publiques à la conception du patrimoine naturel qu'elle véhicule. Nous verrons ensuite que si cet outil d'aide à décision présente tous les attributs de la ressource d'autorité, il apparaît assez peu mobilisé directement par les pouvoirs publics. Cela nous amènera enfin à proposer une typologie de la participation disparate de l'évaluation à la petite fabrique des valeurs du patrimoine naturel.

\section{L'évaluation économique de l'environnement, une démarche orientée vers l'aide à la décision}

\section{1 Évaluation économique et décision publique, le renouveau d'une histoire déjà ancienne}

L'idée de recourir à des analyses coût-bénéfices ${ }^{2}$ (ACB) afin de rationaliser les décisions publiques en matière d'environnement en identifiant la « meilleure » alternative possible parmi plusieurs options (Pearce et al., 2006) n'est pas nouvelle. Dès 1808, Gallatin, Secrétaire d'État au Trésor américain, recommandait son emploi pour les projets liés à l'eau, secteur dans lequel elle a d'ailleurs été institutionnalisée en 1936 (Flood Control Act). Si les observateurs s'accordent

2 L'ACB vise à décrire les conséquences positives (" bénéfices ») et négatives (" coûts ») d'un projet, à les quantifier monétairement, puis à les agréger afin de les comparer sur une période de temps donnée. La règle de décision généralement prescrite veut que le projet soit mis en œuvre si sa valeur nette actualisée est positive, ou, en cas de comparaison entre scénarios, que celui dont le bénéfice net actualisé est le plus élevé soit choisi. L'ACB trouve ses fondements dans I'analyse micro-économique des préférences (Pearce et al., 2006). 
à considérer que la première estimation de la valeur de la biodiversité a été menée en 1926 en Louisiane (Colon et al., 2009), ces techniques se sont surtout développées ces dernières décennies notamment via le National Environmental Policy Act (1969) obligeant à la réalisation d'études d'impact environnemental et le Presidential Executive Order 12291 (1981) permettant d'exiger le recours à l'ACB lors de la définition des politiques environnementales, mais aussi d'intenter plus facilement des poursuites en cas de préjudice. Les débats ayant suivi le naufrage de l'Exxon-Valdez en 1989, à un moment charnière de l'essor des préoccupations environnementales, ont fortement participé à la légitimation de l'évaluation économique aux États-Unis mais aussi au sein des institutions internationales (Portney, 1994). L'Organisation de Coopération et de Développement Économiques (OCDE) adopte dès 1972 le principe du pollueurpayeur et elle préconise en 1991 le recours systématique à l'évaluation pour les politiques environnementales; de son côté, l'Organisation des Nations Unies (ONU) commande en 2000 les travaux du Millenium Ecosytem Assessment visant à estimer les conséquences des changements environnementaux sur le bien-être des individus. La Commission Européenne ne reste pas à l'écart puisqu'elle mobilise ces techniques pour calculer les montants des mesures compensatoires de la Politique Agricole Commune (PAC) et soutient, depuis 2008, l'étude The Economics of Ecosystems and Biodiversity (TEEB).

Malgré ce faisceau convergent d'incitations et de normes, l'évaluation resterait moins prisée en France que dans les pays anglo-saxons (Treich, 2008). L'argument d'un système économique plus réticent, en France, à l'épanouissement du marché libre et de la régulation marchande est souvent avancé. De même, il existerait aux États-Unis un lobby en faveur de l'ACB et des liens beaucoup plus forts entre milieux académiques et politiques (Pearce et al., 2006). Cependant, dès 1977, les décrets réglementaires français relatifs aux installations classées et à la protection de la nature font explicitement référence à l'évaluation. Les lois de finances de 2001 exigent le recours à l'ACB dans les domaines du transport et de l'eau, et celle relative à la révision constitutionnelle de 2009 l'exécution d'études d'impact environnemental. Dès lors, si on peut effectivement considérer que la France a mis un certain temps à s'approprier cette conception de l'action publique, force est de constater qu'elle cherche depuis à la faire sienne. Sous l'impulsion du Conseil d'État, le rapport « Sécurité juridique et complexité du droit » de 2006 note ainsi que « la décision politique [...] ne peut être prise qu'au vu d'une évaluation précise de la situation actuelle et du bilan coûts-avantages, même sommaire, de chacune des options possibles ».

\subsection{Le patrimoine naturel comme extension du champ d'application de l'économie standard}

D'après Godard (2004), le succès récent de l'économie de l'environnement tiendrait au fait que les décideurs ont vu dans son outillage néoclassique la base la plus solide, parmi la contribution possible des sciences sociales, à la rationalisation des processus de décision publique. En effet, cette approche 
des enjeux environnementaux fondée sur les notions d'utilité, de bien-être, de préférences individuelles, mais aussi de priorité à la coordination par les prix, a bénéficié de la puissance de formalisation de la théorie néoclassique et de la masse des chercheurs ayant appliqué dans ce nouveau champ des outils déjà connus et maîtrisés. Cette extension a d'abord porté sur les externalités ${ }^{3}$ liées aux transports, puis dans les années 1980 aux nuisances (pollution atmosphérique, déchets...), avant de se consacrer dans les années 1990 au changement climatique, à la biodiversité et plus généralement au "patrimoine naturel ». La définition de ce dernier comme "capital naturel générateur de services» (Desaigues et Point, 1993) est intéressante puisque ces services, supposés faire le lien entre offre et demande, permettent l'internalisation des externalités. Bien qu'ambiguë car utilisée de manière équivalente à celles de «biens d'environnement » ou «d'actifs naturels », l'expression « patrimoine naturel » aurait néanmoins le mérite d'insister sur l'aspect multifonctionnel des objets environnementaux : « décrire le patrimoine naturel, c'est rendre compte de la nature extrêmement diversifiée de la demande dans ce domaine » (Point, 1998).

Afin de préciser les différentes dimensions de cette demande, une typologie de valeurs est alors élaborée. La première catégorie renvoie aux valeurs d'usages, qu'ils soient directs (consommation), induits (utilisation du bien comme facteur de production) ou indirects (bénéfices tirés d'un changement plus global tel que la régulation du climat). Mais, les individus peuvent aussi, par exemple pour des raisons éthiques ou par altruisme, attribuer à un bien des valeurs dites de non-usage : conservation pour les héritiers (valeur de legs), pour soi dans le futur (valeur d'option) ou pour que d'autres puissent en bénéficier (valeur de disponibilité pour les contemporains). La valeur intrinsèque quant à elle provient de la nature même du bien, de sa simple existence. Enfin, la valeur de quasioption se présente lorsqu'un investissement est irréversible et ses bénéfices futurs inconnus, les agents étant susceptibles de préférer différer la mise en œuvre du projet. La Valeur Économique Totale (VET) du patrimoine naturel est alors formée de l'ensemble de ces valeurs (Figure 1).

Différentes approches peuvent être mobilisées pour estimer ces valeurs. Les méthodes de révélation des préférences (coûts de déplacement et prix hédonistes ${ }^{4}$ ) consistent à étudier le marché existant d'un bien lié à celui dont on cherche la valeur. Les méthodes d'observation des préférences déclarées (évaluation

3 Une externalité est une situation dans laquelle un agent économique a, par son activité de consommation ou de production, une influence positive ou négative sur un autre agent, sans qu'il n'y ait de compensation monétaire de ces dommages ou bénéfices.

4 La méthode des coûts de déplacement utilise les dépenses engagées par un individu pour se rendre sur un site (récréatif notamment) comme un indicateur de son consentement-à-payer pour cette visite (Clawson et Knetsch, 1966). La méthode des prix hédonistes est quant à elle basée sur l'idée que le prix d'un bien hétérogène dépend de ses caractéristiques propres, mais aussi de critères environnementaux (Rosen, 1974). Un exemple parlant est celui des biens immobiliers dont le prix varie selon qu'ils offrent ou non une vue sur mer. 


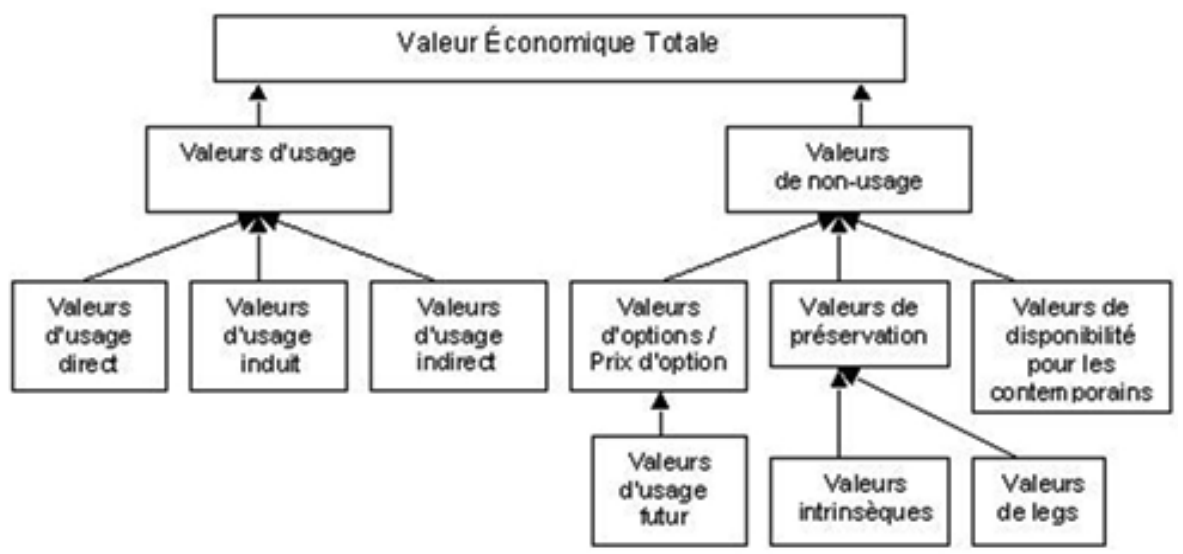

Fig. 1 Décomposition de la VET (d'après Point (1998))

The components of the total economic value (from Point (1998))

contingente 5 (MEC) notamment) reconstituent par voie d'enquête un marché fictif et interrogent, selon une technique adaptée, les usagers sur la valeur qu'ils accordent au bien (Desaigues et Point, 1993). Les secondes permettent d'estimer l'ensemble des valeurs, au contraire des premières qui n'appréhendent que les valeurs d'usage. Elles seraient donc plus adaptées au patrimoine naturel pour lequel les valeurs de non-usage se révèlent souvent importantes, et permettent en outre de s'intéresser à des situations hypothétiques. Précisons néanmoins que ces ajustements méthodologiques peinent à convaincre ceux qui considèrent comme vaine et dénuée de sens l'idée même d'essayer de déterminer une valeur globale en additionnant des éléments quantifiables et des éléments relevant de l'éthique ou de l'esthétique (Harribey, 1997).

\section{L'évaluation économique ou le savoir d'experts comme ressource d'autorité ?}

\subsection{Une certaine conception de la décision}

Le cadre théorique de l'ACB suppose un décideur public bienveillant et omniscient agissant au nom de la société afin d'identifier la politique la plus efficiente (Baumstark, 2009). Cette vision laisse a priori peu de place à la concertation, et ce d'autant plus que l'application de l'ACB favoriserait les modes de pensées et les valeurs des acteurs dominants (Roy et Damart, 2002). Ainsi, les évaluations conduites par les services de l'État sont non seulement les plus diffusées (Cohen

5 La MEC vise, sur la base de scénarios hypothétiques proposant une description de l'évolution de la qualité de l'environnement et y associant un mode de paiement, à faire révéler leur valorisation aux personnes interrogées (Mitchell et Carson, 1989 ; Arrow et al., 1993 ; Desaigues et Point, 1993). 
de Lara et Dron, 1997) mais leur objet est de plus fortement influencé par les programmes de recherche, donc par les pouvoirs publics (Point, 1998). Par exemple, les effets des politiques de transport sur le désenclavement local ont été beaucoup moins étudiés que leurs conséquences sur la santé (Cohen de Lara et Dron, 1997).

Une autre raison avancée pour expliquer cette relation ambiguë entre évaluation et pouvoir tient au fait que les méthodes seraient peu accessibles et leurs résultats opaques pour des utilisateurs souvent peu familiers de la démarche. Cette complexité renforcerait le caractère technocratique de la décision (Roy et Damart, 2002), les risques de manipulation (Cohen de Lara et Dron, 1997) et de confiscation des débats (Baumstark, 2009). Dans un certain nombre d'ACB, l'évaluation devient ainsi une "norme qui consacre à la fois la valeur du bien public considéré et l'efficacité de l'action publique » (Dujin et al., 2008). Le chiffre est souhaité pour lui-même (Milanesi, 2010) et généralement présenté "sinon comme exact, du moins comme le reflet d'une réalité objective " (Roy et Damart, 2002). De fait, les valeurs sont souvent reprises sans que les méthodes de calcul, leurs fondements théoriques et hypothèses ne soient explicités (Milanesi, 2010) ; cet aspect a même fait l'objet d'articles aux titres provocateurs tel "Contingent Valuation: Is some number better than no number? » de Diamond et Hausman (1994).

Le cas du patrimoine naturel illustre de manière particulièrement vive ces ambiguïtés car, alors qu'elle repose, du moins en théorie, sur la prise en compte d'une diversité d'usages, la VET tendrait à masquer et réduire la pluralité des ordres de justification. Plottu et Plottu (2010) notent ainsi qu'elle ne permet pas de saisir « la partie cachée de la valeur [...] liée à la notion de patrimoine, à la valeur symbolique et identitaire [...] qui permet à une communauté sur un territoire de se reconnaître comme une ». Par l'adéquation du référentiel monétaire au langage des décideurs, la tautologie de ses présupposés théoriques mais également les non-dits de sa démarche, l'évaluation pourrait donc avoir acquis une certaine capacité à coloniser et phagocyter les procédures de choix publics, allant même, pour certains, jusqu'à se substituer aux processus démocratiques (Milanesi, 2010).

\subsection{Faire accepter une vision prédéfinie de l'intérêt général}

Censée être utilisée ex-ante afin de rationaliser la décision publique en comparant différentes stratégies d'allocations des ressources rares, l'ACB ne considère souvent dans les faits qu'une seule option. Les conclusions sont alors susceptibles d'être instrumentalisées afin de valider une décision déjà prise. Cet extrait d'Ayoun et al. (2009) est particulièrement éclairant : "Dans le processus d'élaboration des contrats de la Région Île-de-France, [...] le calcul socio-économique, qui est utilisé dans la phase d'évaluation de l'ensemble des opérations prévues dans le contrat du projet, ne l'est qu'au titre d'un élément permettant de vérifier que [le projet] est socio-économiquement dans la norme. Ce calcul ne sert donc pas à hiérarchiser des options - ces choix ont été faits auparavant. " Par ailleurs, même lorsqu'il s'agit de choisir entre plusieurs projets, la légitimité de leur réalisation 
n'est que rarement questionnée (Cambou et al., 2009). C'est un des nombreux reproches adressés à la Commission Roskill visant à choisir le site d'implantation d'un nouvel aéroport à Londres en 1971 : elle ne posait pas la question de la nécessité de cette nouvelle infrastructure (Moindrot et Kleinbauer, 1972).

L'ACB peut aussi servir à légitimer les dépenses engagées (Pearce et al., 2006) en étant alors mobilisée ex-post. Il s'agit de «valider, par la sanction de la demande sociale, le bien-fondé du financement» (Maresca et al., 2006). Les estimations de la valeur des récifs coralliens dans l'archipel de Vanuatu furent ainsi utilisées par les banques de développement pour mettre en évidence les conséquences positives des politiques de conservation existantes pour les économies locales (Laurans et al., 2013b). De même, l'Agence de l'Eau Seine Normandie a, en 1995, simultanément augmenté les redevances et financé des programmes de protection des zones humides puis réalisé une évaluation afin de justifier a posteriori ces investissements (Colon et al., 2009). Enfin, même s'il est encore un peu tôt pour juger, le cas des Parcs Nationaux est également intéressant. La réforme de 2006 donne la possibilité aux communes situées notamment dans l'ancienne zone périphérique d'accepter ou non d'adhérer à la nouvelle charte du Parc. Il existe donc un risque de voir certaines communes se retirer du projet. Une note de synthèse réalisée en 2008 note que «les établissements sont donc confrontés à la nécessité de convaincre les communes d'une part de la qualité de leur projet et d'autre part de l'intérêt de faire partie du territoire $d u$ parc. Pour cela il convient d'élaborer des argumentaires assis, autant que faire se peut, sur des études, des enquêtes et des expériences scientifiquement validées et de mettre en évidence la plus-value apportée par l'existence des parcs à leur territoire : retombées économiques (flux économiques, emplois...) mais aussi éléments de valeur et services écologiques » (Parcs Nationaux de France, 2008, souligné par les auteurs). Autrement dit, il ne s'agit pas tant de faire accepter la protection la nature en ce qu'elle contient un potentiel économique (Depraz, 2008), que de montrer a posteriori les bienfaits qu'elle a pu générer. Dans cette optique, des «préconisations pour évaluer la valeur des Parcs » sont également mises à disposition des établissements et force est de constater que les méthodes d'évaluation occupent une place significative parmi les outils économiques proposés. Mais, cette utilisation ex post des évaluations peut aussi se retourner contre leurs auteurs et les méthodes mobilisées, notamment lorsqu'elles conduisent à invalider la décision politique anciennement prise (De Rus et Inglada, 1997).

\subsection{Une ressource d'autorité finalement peu efficace pour les pouvoirs publics?}

Malgré ses facultés supposées et bien qu'elle soit fortement recommandée dans les textes réglementaires, l'évaluation resterait paradoxalement assez peu utilisée en appui direct des politiques publiques (Cohen de Lara et Dron, 1997). Dans les Caraïbes, des estimations de la valeur des écosystèmes marins sont pourtant à l'origine d'un certain nombre de projets, tels que la création d'un parc sur l'île 
de Bonaire, l'augmentation des droits d'entrée sur une réserve en République dominicaine ou encore l'adoption de mesures de protection d'une partie du littoral des Bahamas. Mais, Kushner et al. (2012) relèvent que seules 13 de ces études eurent une influence sur la décision et ce alors même qu'ils en recensent plus de 200. En fait, pour les auteurs, la transformation de ces évaluations en programmes d'action tiendrait notamment au degré de dépendance de l'économie nationale aux écosystèmes à protéger. Ainsi, les résultats des évaluations furent plus suivis à Bonaire, dont l'économie est fortement tributaire des plongées touristiques, qu'à Trinidad et Tobago qui dispose de ressources pétrolières et gazières importantes.

Au-delà de ces configurations très particulières, il semble cependant que les décisions concernant les politiques environnementales soient souvent fondées sur des bénéfices espérés mais non évalués, $i$. e. sur une évaluation "intuitive et politique» (Baumstark, 2009). Parmi les raisons invoquées, la méfiance à l'égard de la démarche occupe une place importante, qu'elle concerne la manière d'appréhender le patrimoine naturel (Colon et al., 2009) ou des résultats jugés d'autant plus partiaux qu'ils émanent souvent du maitre d'ouvrage (Cambou et al., 2009). De même, la monétarisation semble tout autant une force qu'une faiblesse au regard des connotations négatives qu'elle véhicule, notamment dans le domaine du patrimoine naturel puisque c'est à l'ordre marchand que de nombreux groupes attribuent les responsabilités des maux environnementaux (Pearce et al., 2006).

En outre, même lorsque l'évaluation est commandée, ses recommandations semblent peu suivies par les pouvoirs publics en raison des «écarts importants entre les résultats $[\ldots]$ et les attentes des décideurs et, plus généralement encore, les attentes de la société »(Baumstark, 2009). Par exemple, les effets de coupure, pourtant cruciaux en termes de consommation d'espaces, sont rarement intégrés aux ACB réalisées en France dans le domaine des transports, en raison des difficultés méthodologiques qu'ils impliquent (Héran, 2009). Ces contraintes pourraient également expliquer pourquoi, sur l'ensemble des évaluations environnementales réalisées depuis 2002 en France, une forte proportion se focalise sur les activités récréatives (Mahieu et al., à paraître), au détriment d'autres services écosystémiques moins facile à monétariser. Au final, les pouvoirs publics paraissent hésiter à mettre en œuvre des politiques fondées sur la seule justification d'une allocation efficiente des ressources, notamment lorsqu'elles vont à l'encontre des réalités sociales et des singularités territoriales (Faburel, 2009). Des projets dont l'arbitrage coûts-avantages est remis en question par les ACB, comme le canal Rhin-Rhône, peuvent ainsi être soutenus politiquement. À l'inverse, Garrod et Willis (1996) notent que le gouvernement anglais a bloqué un projet visant à lutter contre la baisse du débit de la Darent, et cela alors même qu'une étude avait montré que sa valeur nette actualisée était positive. Les auteurs supposent que les pouvoirs publics se sont questionnés sur l'estimation des valeurs de non-usage effectuée et qui représentaient $90 \%$ des bénéfices escomptés. Ces différents exemples tendraient donc à confirmer que les logiques de l'évaluation ne 
resteraient qu'une « une solution technique à un problème politique » (Facchini, 1994).

Enfin, au-delà de ces doutes, il est nécessaire de prendre en compte la transformation des modalités même de l'action publique. Pour Godard (2004), ce type ce langage aurait ainsi perdu de son effectivité sociale à mesure que les rapports entre l'État et la société civile évoluaient suite à une plus grande implication des parties prenantes. Les associations de riverains et élus utilisèrent notamment l'enjeu du crapaud "sonneur à ventre jaune " (espèce protégée) pour dénoncer l'absence d'une prise en compte satisfaisante des conséquences environnementales de la portion Lyon-Balbigny de l'A89, entraînant le report de sa mise en service de 1989 à 2013. Ceci n'est qu'un des nombreux exemples de l'emploi contre-productif de l'évaluation comme outil visant à passer en force dans le domaine de l'aménagement (Colon et al., 2009). La multiplication des acteurs a une autre conséquence plus inattendue mais tout aussi négative sur le rôle des évaluations comme outil d'aide à la décision : certains décisionnaires craignent, en révélant leurs préférences via des $\mathrm{ACB}$, de perdre leurs capacités à naviguer entre les positions en présence (Kuik, 1992).

\section{La petite fabrique des valeurs}

Si la première partie de l'état des lieux invite à relativiser fortement l'idée d'une utilisation de l'évaluation économique comme ressources d'autorité des politiques de protection du patrimoine naturel, notamment en France, il serait sans doute fallacieux d'en conclure que ces démarches demeurent sans effet. Portés ou instrumentalisés, à différentes échelles et sur différents registres par des acteurs autres que les pouvoirs publics, leurs usages semblent au contraire s'être adaptés à la vitalité et à la complexité d'un jeu patrimonial qui, articulant de multiples «mondes» (institutionnels, scientifiques, économiques), demeure un champ stratégique pour conserver ou transformer les rapports de forces. Il s'agit donc maintenant de regarder comment cet outil permet à certains acteurs d'imposer leurs points de vue et comment, dans certains cas, il est possible de le lire comme " une histoire et un processus de construction de valeurs, d'accord et de désaccord, de négociation, voire d'affrontements » (Laurans et al., 2001) qui participent à injecter de la pluralité dans l'ordre patrimonial.

\subsection{De la sensibilisation au lobbying}

Classiquement, l'évaluation est tout d'abord utilisée afin de sensibiliser, "changer [le] regard [... en] montrant que l'on ne doit pas percevoir [l'environnement] uniquement comme un capital que l'on consomme mais [aussi] comme un capital "classique" dont on ne consommerait que les intérêts » (Colon et al., 2009). Ce rôle est explicite dans le projet TEEB visant à mettre l'accent sur les bénéfices de la biodiversité et sur les coûts liés à sa dégradation. Les estimations issues du 
UK National Ecosytem Assessment, relayées massivement par les médias ${ }^{6}$ et les

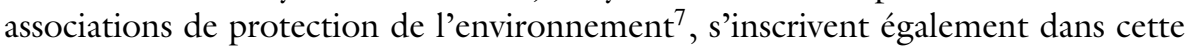
optique. Mais l'exemple le plus saisissant est l'estimation de la valeur des services écosystémiques mondiaux comprise entre 16000 et 54000 milliards \$ fournie par Costanza et al. (1997). Par ce chiffrage spectaculaire et sa comparaison avec le PIB annuel moyen de 18000 milliards \$, l'objectif est de "mettre en avant une valeur qui soit suffisamment importante pour provoquer un électrochoc parmi les décideurs »(Chevassus-au-Louis et al., 2009). S'il est difficile de cerner ses conséquences réelles, on peut noter que cette valeur fut reprise, entre autres, par WWF. De fait, les grandes organisations non gouvernementales (ONG) environnementales jouent un rôle de plus en plus important dans cette instrumentalisation des évaluations, le but étant, du moins dans un premier temps, de «faire connaitre et reconnaitre » (WWF, 2011) afin de s'attirer les faveurs du public et d'interpeller les États. Dans cette perspective, les valeurs sont souvent reprises et mises en récit à une échelle globale (et dans une moindre mesure nationale). Ce type d'instrumentalisation répond aux modes d'actions et aux échelles de légitimités des grands organismes, mais se nourrit également de l'idée très discutable, et pourtant assez répandue, que la crédibilité d'une évaluation dépend de son degré de généralité supposée (Godard, 2004). Les estimations de Costanza et al. (1997) sont ainsi issues de l'extrapolation et de la compilation d'études réalisées par d'autres. Or, pour des éléments du patrimoine naturel comme la biodiversité, les spécificités locales sont cruciales (Pearce et al., 2006) et l'extrapolation entraîne une généralisation abusive. Précisons que ce travail de sensibilisation s'accommode souvent très bien du manque d'explication des méthodes et hypothèses qui confère aux chiffres un statut d'objectivité et une autorité difficile à remettre en question.

À ce niveau, la frontière entre sensibilisation et lobbying devient particulièrement floue. D'ailleurs, certains acteurs n'hésitent pas à franchir le pas et à adopter le langage de l'évaluation, non plus seulement pour sensibiliser, mais pour obtenir une subvention, un accord ou la mise en ouvre d'un programme en influençant directement la décision publique. Coral Reef Initiatives for the Pacific note ainsi que "peu sensibles aux arguments élaborés par les scientifiques, [...] les décideurs ne sont prêts à adhérer aux thèses des protecteurs des récifs $[\ldots]$ qu'à la condition que la conservation ne leur coûte pas cher et n'engendre aucun conflit avec d'autres usagers, électeurs potentiels ou administrés [...] En attribuant une valeur monétaire au récif, on intègre l'écologie dans la sphère économique et on adresse aux décideurs un message dans un langage qu'ils comprennent, changeant ainsi la nature de leur décision» (David et al., 2007). Selon Lal (2004), le moratoire visant à protéger les mangroves aux Îles Fidji (1983) n’a ainsi été signé que parce que leur valeur, estimée à l'initiative d'une

6 e. g. les articles publiés sur les sites de la BBC le 01/06/2011 ou de la Tribune le 06/06/2011. Accès le 24/11/2011.

7 e. g. la réaction publiée sur le site de WWF le 02/06/2011. Accès le 24/11/2011. 
ONG, a convaincu le Ministère de tutelle de l'intérêt de les préserver. De fait, les grandes associations environnementales ne se contentent plus simplement de « consommer» des évaluations mais deviennent productrices d'expertises. En ce sens, le développement de ces outils contribue à renforcer leurs positions dans « le champ de bataille» de la conservation (Millian et Rodary, 2010). Le titre d'une étude réalisée par WWF en Afrique du Sud (2007) apparait ici tout à fait symptomatique : Using valuations to influence decision-making: The economic value of marine protected areas along the Garden Route coast. Ces exemples conduisent donc à nuancer l'idée selon laquelle pouvoirs publics et associations environnementales apprécieraient différemment la légitimité sociopolitique de l'évaluation selon leur représentation et leur rôle dans le fonctionnement des espaces démocratiques (Pearce et al., 2006 ; Faburel, 2009). Elle reste ici utilisée afin d'emporter la décision au risque de remplacer le contrôle de l'État par un «éco-pouvoir " (Lascoumes, 1994) basé sur la capacité de certains groupes à mobiliser et maîtriser des outils de l'expertise scientifique (Amelot et al., 2011).

Mais, ce travail de lobbying n'est pas uniquement réservé aux promoteurs de la cause environnementale. Une utilisation stratégique de l'évaluation peut également permettre à des acteurs économiques de transformer des contraintes apparentes en nouveaux registres de légitimités. À cet égard, le cas du massif forestier des Landes de Gascogne, où l'accentuation des processus de patrimonialisation et d'écologisation contribue à alimenter la tension entre patrimoine privé (la propriété forestière) et patrimoine commun (la forêt comme archétype de nature et réserve de biodiversité), est intéressant. Engagés avant tout dans une logique de production, les sylviculteurs landais se trouvent confrontés à une multiplication de zonages environnementaux, plus ou moins prescriptifs (arrêtés de biotope, zones Natura 2000, ZNIEFF...), à une fréquentation conséquente (Dehez, 2012) et à une certaine remise en cause de leurs pratiques (Deuffic et al., 2010). Mais, les organisations professionnelles disposent également d'un certain nombre de ressources institutionnelles et d'arguments pour faire prévaloir leur propre interprétation de cette demande sociale. La publication de l'estimation monétaire des services éco-systémiques fournis par la forêt française (Chevassusau-Louis et al., 2009) en fait désormais partie. La valeur de $970 €$ par hectare et par an se trouve ainsi régulièrement brandie, aussi bien auprès des pouvoirs publics que dans les médias ${ }^{8}$, sans que son mode de calcul et les hypothèses retenues ne soient véritablement repris et explicités par les utilisateurs. Pourtant, ce chiffre est une estimation moyenne (avec une fourchette pouvant varier de 500 à $2000 € / \mathrm{ha} / \mathrm{an}$ ) obtenue, qui plus est, à partir de la compilation de différentes études portant sur « les forêts françaises tempérées » (Chevassus-au-Louis et al., 2009). Son utilisation in extenso dans le cas particulier d'une forêt cultivée et mono-spécifique de pins maritimes pourrait donc être questionnée. Or, loin de susciter des débats, ce résultat semble davantage jouer le rôle d'un argument

8 e. g. I'article "Incendie de Lacanau : "Le mégot de trop" » publié par Sud-Ouest le 01/09/2012. Accès le 24/09/2012. http://www.sudouest.fr/2012/09/01/lacanau-le-megot-de-trop-808912-706.php 
d'autorité permettant aux forestiers de rappeler les services sociaux et culturels qu'ils rendent à la collectivité (forêt privée en accès libre et gratuit) mais également de revendiquer, via les fonctions environnementales évaluées (notamment le stockage carbone), de nouveaux domaines et échelles de légitimités (Pottier, 2012).

\subsection{Un outil de négociation, entre contestation et reconnaissance}

Il serait toutefois erroné de considérer que les évaluations ne servent qu'à renforcer le pouvoir de certains acteurs et leur influence au sein de structures informelles de décisions associant élites et experts. En s'intéressant à des projets d'aménagement des rivières anglaises, Henry (1984) a notamment montré comment des associations environnementales locales ont adopté les possibilités de la microéconomie comme langage de contestation publique et instrument de négociation. Dans cette configuration, l'évaluation est plutôt appliquée localement, mais reste souvent menée par des experts, même si les enjeux conflictuels peuvent s'accompagner d'une plus grande exigence des parties prenantes en termes de clarté des méthodes et des résultats. De manière identique, les opposants au projet de l'aéroport de Notre-Dames-des-Landes (NDL) ont remis en cause les résultats de l'enquête d'utilité publique conduite en 2006 en faisant réaliser une autre estimation par «un cabinet d'étude européen indépendant ${ }^{9} »$. Selon ses initiateurs, cette dernière montre que «le [coût du] projet pourrait se chiffrer à plus de 600 millions $€$ alors que le dossier d'enquête publique montrait un bénéfice global de plusieurs centaines de milliers d'euros ». Au-delà de cette bataille de chiffres, tout de même révélatrice d'une certaine forme de banalisation de la rhétorique "utilitariste ", il est surtout intéressant de voir comment l'évaluation permet aux opposants d'engager le débat sur les éléments environnementaux qui méritent ou non d'être intégrés au calcul : « [Dans l'enquête d'utilité publique] le coût environnemental est limité aux déplacements terrestres et aux émissions des transports aériens! Aucune estimation n'est faite du coût de la gestion de l'eau (risques d'inondation), des coûts de compensation de la suppression ou de la dégradation des zones humides, de la valeur des services rendus par la nature (par exemple la capture du carbone par la zone de NDL peut être estimée à 10,50 M€). »

Les catastrophes écologiques apparaissent comme un autre moment privilégié de l'utilisation de l'évaluation dans le but de faire reconnaître l'existence des multiples valeurs du patrimoine naturel. Le procès engagé suite au naufrage de l'Exxon-Valdez a ainsi reconnu la possibilité d'obtenir réparation pour des pertes d'usage, mais aussi de non-usage. Il a également donné lieu à d'importantes discussions quant au montant de l'indemnisation, controverse qui a conduit la National Oceanic and Atmospheric Administration à commanditer un guide de

9 Etude disponible sur le site internet de la Confédération paysanne au lien suivant : http://www.confederationpaysanne-pdl.fr/IMG/pdf/4pages-cedpa-resultats-etude-oct11-NB.pdf. Accès le 02/04/2014. 
bonnes pratiques en MEC (Arrow et al., 1993). En France, au moment du naufrage de l'Erika (1999), la loi ne reconnaissait que les préjudices moraux et matériels liés aux coûts de remise en état ou de nettoyage et seuls les services écosystémiques marchands (conchyliculture, fréquentation touristique...) pouvaient être indemnisés. Or, l'évaluation commandée par l'Association interrégionale Ouest Littoral Solidaire, partie civile au procès, évoque le principe de "préjudice écologique " (environ 370 millions $€$ ). Pour appréhender cette notion, elle intègre à la fois des usages liés à la sphère marchande (loisirs) mais aussi les pertes d'agréments des résidents des trois régions concernés (Bretagne, Loire-Atlantique et Poitou-Charentes) et les dommages subis, en l'absence de tout usage, du fait de la marée noire. Ces derniers sont évalués notamment à partir de ce que les habitants sont prêts à payer pour qu'une catastrophe similaire ne se reproduise pas, mais aussi des dépenses de protection et de conservation du littoral engagées par les collectivités et les associations (Bonnieux, 2006). Après plusieurs années de débats, la justice a reconnu, en 2010, l'existence d'un "préjudice écologique résultant d'une atteinte aux actifs environnementaux non marchands, réparables par équivalent monétaire » et accordé aux parties civiles 200 millions $€$ de dommages et intérêts, dont environ 13 millions $€$ au titre du préjudice écologique. C'est ainsi que la Ligue de Protection des Oiseaux fut dédommagée à hauteur de $300000 €$. En 2013, une proposition de loi visant à inscrire le principe de "préjudice écologique » dans le code civil a été adoptée à l'unanimité par le Sénat. Bien que tout à fait exceptionnel, cet exemple montre la contribution d'un travail d'évaluation à une petite révolution dans le domaine du droit de l'environnement.

\subsection{De la coordination à la co-construction des valeurs}

Passer de la négociation et de la reconnaissance à la co-construction suppose de concevoir l'évaluation non plus comme un outil permettant de trouver un accord général sur des valeurs normalisées, mais comme un instrument de connaissance et délibération prenant appui sur un sous-ensemble d'objets et d'actions que les représentants de chaque ordre peuvent discuter (Godard, 1990). Autrement dit, dans cette configuration où l'évaluation est menée en réponse à une problématique et dans un contexte territorial spécifique, ce sont moins les résultats que la construction du dispositif qui importe. De telles démarches sont plus rares et se distinguent par leurs modalités et finalités de mise en œuvre, mais l'un de leurs principaux points communs est qu'elles ouvrent l'expertise à des non-experts.

L'évaluation peut ainsi être mobilisée afin de contribuer à l'identification de l'ensemble des acteurs concernés par un projet et participer à la reconnaissance des pluralités engagées dans ce processus collectif. L'inventaire des usages, des objets et des ressources (Pearce et al., 2006) mais aussi des interdépendances entre écosystèmes et usagers (Kushner et al., 2012) constitue en effet un moyen efficace de recenser les différents groupes d'acteurs en présence, même les plus « petits» et les plus difficilement accessibles (groupes sociaux marginalisés, résidents secondaires...). Alors que ces derniers sont souvent absents de la scène 
politique, l'évaluation peut aider à prendre en compte leurs intérêts dans le processus de décision. Tout en partant d'études territorialisées, cette procédure contribuerait donc à réinscrire les contextes locaux dans une communauté plus large d'usagers (Godard, 1999). L'évaluation peut aussi permettre de reconnaître les efforts consentis par chaque partie et les transferts garantissant l'équité de la politique (Bommelaer et al., 2010). En s'appuyant sur une expérience menée dans le cadre de l'élaboration de Schémas Directeurs d'Aménagement et de Gestion des Eaux (SDAGE), Laurans et al. (2001) montrent ainsi que si les résultats n'ont pas modifié les votes lors des assemblées, la co-construction de l'évaluation a mis en lumière les enjeux des politiques discutées pour chaque catégorie d'acteurs et les transferts de revenu ou d'avantages occasionnés.

Mais cette co-construction des valeurs suppose un ajustement des méthodes afin que la décision ne dépende plus uniquement des experts et/ou gestionnaires (Dujin et al., 2008) et qu'elle perde de sa dimension technocratique (Treich, 2008). Dans cette perspective, l'estimation des préférences déclarées reposant sur des enquêtes auprès des usagers, apparait préférable. Bouteloup (2008) montre par exemple que, dans le cas de l'Erika, mis à part des cas assez rares de rejet pur et simple de la monétarisation environnementale, les parties civiles ont exprimé deux attentes : certains souhaitaient un « chiffrage de base » compréhensible par tous et les autres cherchaient une évaluation globale des dommages, en cohérence avec des principes moraux. Ces derniers préféraient alors l'évaluation contingente (MEC) aux estimations planchers en estimant que cette approche leur ouvrait un espace de participation et d'expression directe. L'un des principaux enjeux de l'évaluation n'est en conséquence pas tant de résoudre les conflits d'intérêts qui structurent les problèmes environnementaux mais au contraire, de faire en sorte qu'ils puissent s'exprimer à travers elle. La méthode des choix multi-attributs ${ }^{10}$, de plus en plus mobilisée, présente ainsi l'avantage de proposer à l'enquêté un arbitrage entre différentes politiques (Maresca et al., 2006). Elle suppose, tout comme la MEC dont elle est un prolongement, la mise en place de focus groups, d'entretiens auprès des acteurs clés ou de pré-enquête auprès des populations concernées afin de construire des scénarios traduisant la diversité des points de vue. Des conflits et négociations peuvent alors apparaitre à cette étape, déplaçant en quelque sorte la contestation en amont de la décision (Carias Vega et Alpizar, 2011). Si des biais sociologiques importants peuvent altérer la sélection initiale des scénarios soumis à la discussion collective, le caractère concerté de l'élaboration des choix effectivement proposés lors de l'enquête et la qualité de l'animation constituent la réelle plus-value en direction d'une co-construction.

10 Fondée sur la théorie des caractéristiques de Lancaster (1966), elle considère que la satisfaction que les individus retirent de la consommation d'un bien provient en fait de la consommation des différentes caractéristiques qui le composent (Louviere et al., 2000 ; Bennett et Blamey, 2001 ; Hanley et al. 2001). 


\section{Conclusion}

Présente depuis longtemps dans les pays anglo-saxons, relayée ces dernières décennies par les organismes internationaux, l'évaluation économique s'est progressivement déployée dans le paysage institutionnel français en appui notamment des politiques environnementales. Considérer la nature, non comme un objet original, mais comme une simple extension des champs d'application de la théorie néoclassique semble avoir paradoxalement favorisé ce processus d'institutionnalisation malgré toutes les ambiguïtés d'une telle posture (Godard, 2004). Dans un contexte de dégradation des ressources naturelles et de réduction des dépenses, la mobilisation d'un savoir scientifique directement applicable et conçu dans un souci de rationalisation et d'efficacité économique de l'action publique était de nature à séduire les pouvoirs publics. L'opacité et la force de persuasion des méthodes d'évaluation purent également constituer des atouts supplémentaires pour des administrations soucieuses d'acheter une forme de paix sociale et de faire accepter une conception prédéfinie de l'intérêt général (Godard, 1990). Mais, même s'il est nécessaire de rester prudents en raison du manque de recul et de l'étendue du champ d'investigation, notre étude tend à nuancer fortement cette utilisation des évaluations comme principe privilégié des arbitrages de l'action publique, notamment en France. Les politiques et projets environnementaux semblent aujourd'hui davantage basés sur un compromis entre intérêts marchands et patrimoniaux, et ce même dans le domaine de l'eau pourtant présenté comme le premier champ d'application de l'économie du bien-être avec l'institution d'un système de redevances sur la pollution et les prélèvements en 1964 (Vivien, 2009).

Mais ce constat n'atténue en rien l'intérêt de suivre le cheminement des méthodes d'évaluation à travers l'espace social, bien au contraire. La diversification des usages stratégiques d'un calcul économique devenu un moyen parmi d'autres d'alimenter la décision (Laurans et al., 2001) constitue un bon indicateur de la transformation des logiques de conservation de la nature et de ses jeux d'acteurs. Suivre les traces de l'instrumentalisation sociale de ces démarches, ou plutôt bien souvent de leurs simples résultats, permet notamment de mettre en lumière le déplacement des responsabilités s'opérant entre l'État, la société civile, l'expertise scientifique et les forces du marché pour relever, après d'autres, l'influence croissante de certains groupes d'acteurs. Il peut s'agir des grandes ONG environnementales engagées dans des logiques d' «éco-pouvoir » (Lascoumes, 1994 ; Milian et Rodary, 2010), mais aussi d'acteurs économiques qui trouvent là l'opportunité de transformer les contraintes de la patrimonialisation, si ce n'est en revenus, du moins en nouvelles légitimités (Depraz, 2008). Pour ces différents protagonistes, qui bénéficient de ressources institutionnelles conséquentes et ont acquis l'expertise nécessaire, l'évaluation peut offrir à la fois un moyen de traduire leurs préoccupations en un langage partagé par les décideurs et des arguments d'autorités permettant d'imposer leurs vues aux autres acteurs. Cela n'est pas sans rappeler certaines logiques bien connues des géographes et notamment l'influence 
croissante de la maîtrise des outils géomatiques (de l'acquisition de données à la production de cartes) dans les processus de délimitation et de légitimation des aires protégées (Amelot et al., 2011).

Au-delà de ces formes d'instrumentalisation, où monétarisation et justifications morales du patrimoine trouvent à s'accommoder, certains exemples tendent à montrer que l'évaluation économique peut également parfois servir d'outil de contestation et de reconnaissance. Dans ces configurations, même si elles restent bien souvent conçues dans une logique d'expertise, les évaluations semblent néanmoins permettre d'engager le débat sur ce qui relève ou non du domaine du patrimoine naturel. Ceci permet d'entrevoir des possibilités pour nourrir l'enjeu de la prise en compte des pluralités dans les processus de patrimonialisation. En effet, à condition de les concevoir non pas comme des exercices standardisés servant à révéler une valeur mais, plus modestement, comme des outils transactionnels et de médiation dans un processus social de connaissance et de délibération (Laurans et al., 2001), les évaluations peuvent contribuer à dépasser les rhétoriques holistiques du patrimoine. Dans cette optique, certains attributs de ces méthodes telles que l'identification systématique des acteurs, et donc aussi des absents de la scène de débat (via leur intégration dans des communautés plus larges d'usagers), l'explicitation des éventuels efforts et transferts de revenus pour chaque groupe d'usagers ou encore la capacité à mettre à l'épreuve de la discussion et du jugement différents programmes de gestion (via les focus groups et les scénarios) peuvent se révéler particulièrement intéressants (Laurans et al., 2001). En retour, un travail pluridisciplinaire peut permettre de définir des évaluations mieux adaptées à la problématique traitée et à ses échelles de gestion, d'affiner les hypothèses (économiques mais aussi sociales et politiques) qui sous-tendent les programmes d'actions proposés et, surtout, de mieux prendre en considération des éléments, tels que l'attachement au lieu ou les perceptions, qui bien que subjectifs peuvent avoir plus d'influence sur les estimations que les variables socioéconomiques généralement intégrées à l'analyse (e.g. Rulleau et Rey-Valette, 2013). Facchini (1994) suggère d'ailleurs que l'évaluation économique d'un paysage soit systématiquement précédée par une évaluation non-économique de la qualité de ce dernier, soit en termes écologiques, soit en termes de perceptions sociales. Au final, si le langage de l'évaluation contribue indéniablement à la petite fabrique du patrimoine naturel, il semble également s'ajuster aux grés de ces processus ; l'enjeu restant bien, pour faire droit à la pluralité des légitimités, de réussir à contenir les réductionnismes de l'ordre patrimonial et de la rationalité marchande. 
50 avenue de Verdun

33610 Cestas

vincent.banos@irstea.fr

Bergerie Nationale

78120 Rambouillet

benedicte.rulleau@uvsq.fr

\section{Bibliographie}

Amelot X., Moreau S., Carrière M. (2011), « Des justiciers de la biodiversité aux injustices spatiales. L'exemple de l'extension du réseau des aires protégées à Madagascar », in Blanchon D., Gardin J., Moreau S. (eds), Justice et injustices environnementales, Nanterre, Presses Universitaires de Paris-Ouest, p. 193-214.

Arrow, K., Solow, R., Portney, P.R., Leamer, E.E., Radner, R., Schuman, H. (1993), Report of the NOAA Panel on Contingent Valuation, Washington DC, NOAA, US Department of Commerce, $67 \mathrm{p}$.

Ayoun, P., Chappond, J., Heux, R., Morcheoine, A. (2009), "Les usages et manques actuels des données et méthodes de monétarisation en France », in Chanel, O., Faburel, G. (eds.), L'environnement dans la décision publique : refonder l'évaluation socio-économique pour des politiques de transport plus durables, Paris, Economica, p. 75-85.

Babelon, J.-P., Chastel, A. (1981), «La notion de patrimoine », Revue de I'art, n 49, p. 5-32.

Banos, V. (2008), L'hypothétique construction des lieux ordinaires entre agriculteurs et non-agriculteurs en Dordogne : de l'idéologie patrimoniale à la recherche des échappés du territoire, Thèse de doctorat en Géographie, Université Paris IV-Sorbonne, 516 p.

Banos, V. (2010), "Le patrimoine au risque de son expansion, vers une "naturalisation" des territoires? - Exemple de l'extension spatiale des processus de patrimonialisation en Périgord ", Sud Ouest Européen, n³0, 37-51.

Barrère, C., Barthélémy, D., Nieddu, M., Vivien, F.-D. (eds.) (2005), Réinventer le patrimoine, de la culture à l'économie, une nouvelle pensée du patrimoine?, Paris, L'Harmattan, 337 p.

Baumstark, L. (2009), "Pour un calcul économique comme ferment d'un référentiel partagé : de I'utilité sociale des "valeurs tutélaires" », in Chanel, O., Faburel, G. (eds.), L'environnement dans la décision publique : refonder l'évaluation socio-économique pour des politiques de transport plus durables, Paris, Economica, p. 125-137.

Bennett, J., Blamey, R. (2001), The Choice Modelling Approach to Environmental Valuation, Cheltenham, Edward Elgar, 269 p.

Bommelaer, O., Curri Lemaître, E., Marcus, V. (2010), "Des méthodes de monétarisation à compléter mais opérationnelles», in Pappalardo, M. (éd.), Donner une valeur à l'environnement: la monétarisation, un exercice délicat mais nécessaire, Paris, La Revue du Commissariat Général au Développement Durable, p. 75-77.

Bonnieux, F. (2006), Évaluation économique du préjudice écologique causé par la catastrophe de I'Erika, Rapport commandité par I'Association interrégionale Ouest Littoral Solidaire, Rennes, INRA, $36 \mathrm{p}$.

Bouteloup, C. (2008), Dynamiques de reconnaissance des dommages écologiques : quels apports de I'évaluation économique?, Colloque «Littoral : Subir, Dire, Agir », Lille, avril 2008, 13 p.

Bürgenmeier, B. (2008), Politiques économiques du développement durable, Paris, De Boeck, 288 p. 
Callon, M., Latour, B. (2013), " "Tu ne calculeras pas !" - ou comment systématiser le don et le capital ", in Callon, M., Akrich, M., Dubuisson-Quellier, S., Grandclément, C., Hennion, A., Latour, B., Mallard, A., Méadel, C., Muniesa, F., Rabeharisoa, V. (eds.), Sociologie des agencements marchands, Paris, Presses des Mines, p. 9-41.

Cambou, J., Fourniau, J.-M., Galtier, B., Mignerey, P. (2009), "Les expériences de débats publics : entre utilité et acceptabilité sociales de l'évaluation socio-économique de l'environnement », in Chanel, O., Faburel, G. (eds.), L'environnement dans la décision publique : refonder l'évaluation socio-économique pour des politiques de transport plus durables, Paris, Economica, p. 84-197.

Carias Vega, D., Alpizar, F. (2011), Choice Experiments in Environmental Impact Assessment - The Case of the Toro 3 Hydroelectric Project and the Recreo Verde Tourist Center in Costa Rica, Environment for Development, Discussion Paper Series EfD DP 11-04, Gothenburg, 22 p.

Chevassus-au-Louis, B., Salles, J.-M., Pujol, J.-L. (2009), Approche économique de la biodiversité et des services liés aux écosystèmes - Contribution à la décision publique, Rapport au Premier Ministre, Centre d'analyse stratégique, Paris, La Documentation française, $376 \mathrm{p}$.

Clawson, M., Knetsch, J.-L. (1966), Economics of Outdoor Recreation, Baltimore, Johns Hopkins University Press, $328 \mathrm{p}$.

Cohen de Lara, M., Dron, D. (1997), Évaluation économique et environnement dans les décisions publiques : rapport au ministre de l'environnement, Paris, La Documentation Française, 416 p.

Colon, M., Mattersdorf, G., Pavageau, C. (2009), La place de l'évaluation économique de la biodiversité et des services écosystémiques dans les processus de décision, Rapport d'étudiants commandité par R. Billé, Paris, AgroParisTech ENGREF/IDDRI, 62 p.

Costanza, R., d'Arge, R., de Groot, R., Farber, S., Grasso, M., Hannon, B., Limburg, K., Naeem, S., O'Neill, R.V., Paruelo, J., Raskin, R.G., Sutton, P., van den Belt, M. (1997), "The value of the world's ecosystem services and natural capital », Nature, n³ 387, p. 253-260.

Dardot, P., Laval, C. (2009), La nouvelle raison du monde. Essai sur la société néolibérale, Paris, La Découverte, $498 \mathrm{p}$.

David, G., Herrenschmidt, J.-B., Mirault, E., Thomassin, A. (2007), Valeur sociale et économique des récifs coralliens du Pacifique insulaire, Nouméa, Coral Reef Initiatives for the Pacific, 40 p.

Dehez, J. (éd.), (2012), L'ouverture des forêts au public : un service récréatif, Versailles, Quae, 165 p.

Depraz, S. (2008), Géographie des espaces naturels protégés - Genèse, principes et enjeux territoriaux, Paris, Armand Colin, 320 p.

De Rus, G., Inglada, V. (1997), "Cost-benefit analysis of the high-speed train in Spain », The Annals of Regional Science, $\mathrm{n}^{\circ} 31$, p. 175-188.

Desaigues, B., Point, P. (1993), Économie du patrimoine naturel : La valorisation des bénéfices de protection de l'environnement, Paris, Economica, 317 p.

Deuffic, P., Ginelli, L., Petit, K. (2010), «Patrimoine foncier... et naturel ? Les propriétaires forestiers face à l'écologisation des Landes de Gascogne », Sud-Ouest européen, n 30, 109-121.

Di Méo, G. (1995), "Patrimoine et territoire, une parenté conceptuelle », Espaces et Sociétés, n 78, p. 9-15.

Diamond, P.A., Hausman, J.A. (1994), «Contingent Valuation : Is Some Number Better than No Number?", Journal of Economic Perspectives, n 8, p. 45-64.

Dujin, A., Maresca, B., Mordret, X., Picard, R. (2008), "La valeur économique et sociale des espaces naturels protégés ", Cahier de Recherche, n²47, Paris, CREDOC, 103 p.

Facchini, F. (1994), "L'évaluation du paysage : revue critique de la littérature », Revue d'économie régionale et urbaine, $\mathrm{n}^{\circ} 3,375-401$.

Faburel, G. (2009), "L'évaluation socio-économique comme coordination des valeurs : vers une territorialisation des méthodes appliquées à l'environnement », in Chanel, O., Faburel, G. (eds.), 
L'environnement dans la décision publique : refonder l'évaluation socio-économique pour des politiques de transport plus durables, Paris, Economica, p. 184-197.

Garrod, G.D., Willis, K.G. (1996), «Estimating the Benefits of Environmental Enhancement: A Case Study of the River Darent », Journal of Environmental Planning and Management, $\mathrm{n}^{\circ} 39$, p. 189-204.

Godard, O. (1990), «Environnement, modes de coordination et systèmes de légitimité : analyse de la catégorie de patrimoine naturel », Revue économique, n 41, p. 215-242.

Godard, O. (1999), "L'évaluation environnementale dans les procédures de coordination publique Quelques orientations », Natures, Sciences, Sociétés, n 7, p. 56-57.

Godard, O. (2004), " Autour des conflits à dimension environnementale - Évaluation économique et coordination dans un monde complexe », Cahiers $n^{\circ}$ 12, Paris, École polytechnique/CNRS, 24 p.

Hanley, N., Mourato, S., Wright, R.E. (2001), "Choice modelling approaches : a superior alternative for environmental valuation? ", Journal of Economic Surveys, n 15, p. 435-462.

Harribey, J.-M. (1997), "La prise en compte des ressources naturelles dans le modèle néoclassique d'équilibre général : éléments de critique », Économies et sociétés, n³5, p. 57-70.

Heinich, N. (2009), La fabrique du patrimoine. De la cathédrale à la petite cuillère, Maison des sciences de l'homme, Paris, $288 \mathrm{p}$.

Henry, C. (1984), "La microéconomie comme langage et enjeu de négociation ", Revue économique, $\mathrm{n}^{\circ} 35$, p. 177-197.

Héran, F. (2009), "Consommation d'espace et effet de coupure », in Chanel O., Faburel G. (éd.) : L'environnement dans la décision publique : refonder l'évaluation socio-économique pour des politiques de transport plus durables, Paris, Economica, p. 49-61.

Jeudy, P.-H. (2001), La machinerie patrimoniale, Paris, Circe, 120 p.

Kushner, B., Waite, R., Burke, L., Jungwiwattanaporn, M. (2012), Influence of Coastal Economic Valuations in the Caribbean - Enabling Conditions and Lessons Learned, Washington D.C., World Resources Institute \& Marine Ecosystem Services Partnership, 20 p.

Kuik, O., Navrud, S., Pearce, D. (1992), "Benefit estimation and environmental decision-making », In : Navrud, S. (éd.), Pricing the European Environment, Oslo, Scandinavian University Press, p. 274-287.

Lal, P. (2004), " Coral Reef Use and Management - The Need, Role, and Prospects of Economic Valuation in the Pacific », in Mahfuzuddin, A., Chong, C.K., Herman, C. (eds.), International Consultative Workshop for Economic Valuation and Policy Priorities for Sustainable Management of Coral Reefs, Penang, WorldFish Center, p. 59-78.

Lancaster, K. (1966), "A new approach to consumer theory », Journal of Political Economy, $\mathrm{n}^{\circ} 74$, p. 132-157.

Landel, P.-A., Senil, N. (2009), "Patrimoine et territoire, les nouvelles ressources du développement ", Développement durable et territoires, Dossier 12 : Identités, patrimoines collectifs et développement soutenable, mis en ligne le 20/01/2009.

Lascoumes, P. (1994), L'éco-pouvoir (environnement et politiques), Paris, La Découverte, 318 p.

Laurans, Y., Bouni, C., Courtecuisse, A., Dubien, I., Johannes, B. (2001), "L'évaluation économique de la théorie à la pratique : I'expérience des SDAGE en France », Natures Sciences Sociétés, n 9, p. 17-28.

Laurans, Y., Rankovic, A., Bill, R., Pirard, R., Mermet, L. (2013a), « Use of ecosystem services economic valuation for decision making : questioning a literature blindspot », Journal of Environmental Management, $\mathrm{n}^{\circ} 119$, p. $208-219$.

Laurans, Y., Pascal, N., Binet, T., Brander, L., Clua, E., David, G., Rojat, D., Seidl, A. (2013b), «Economic valuation of ecosystem services from coral reefs in the South Pacific : Taking stock of recent experience », Journal of Environmental Management, $\mathrm{n}^{\circ} 116$, p. 135-144 
Louviere, J., Hensher, D., Swait, J. (2000), Stated Choice Methods : Analysis and Applications, Cambridge, Cambridge University Press, 402 p.

Mahieu, P.-A., Crastes, R., Kristrom, B., Riera, P. (à paraître), « Non-market valuation in France an overview of the research activity », Revue d'Economie Politique.

Maresca, B., Ranvier, M., Dujin, A. (2006), "Valoriser I'action publique - Le "consentement à payer", un outil au service de la LOLF ", Cahier de Recherche, n²24, Paris, CREDOC, 126 p.

Milian, J., Rodary, E. (2010), "La conservation de la biodiversité par les outils de priorisation ", Revue Tiers Monde, $\mathrm{n}^{\circ} 202$, p. 33-56.

Milanesi, J. (2010), «Éthique et évaluation monétaire de l'environnement : la nature est-elle soluble dans I'utilité ? ", VertigO, n 10, mis en ligne le 29/09/2010.

Mitchell, R.C., Carson, R.T. (1989), Using surveys to value public goods : The contingent valuation method, Baltimore, Resources For the Future Press, $81 \mathrm{p}$

Moindrot, C., Kleinbauer, A. (1972), «Chronique britannique », Norois, n 76, p. 765-779.

Parcs Nationaux de France. (2008), Etude des retombées économiques et de la valeur des parcs nationaux, Note de synthèse, Paris, 7 p.

Pearce, D., Atkinson, G., Mourato, S. (2006), Analyse coûts-bénéfices et environnement-Développements récents, Paris, OCDE, $355 \mathrm{p}$.

Pecqueur, B. (2002), «Dans quelles conditions les objets patrimoniaux peuvent-ils être support d'activités? ", Montagnes Méditerranéennes, n 15, p. 123-129.

Plottu, E., Plottu, B. (2010), «Multidimensionnalité des enjeux du paysage : de l'évaluation à la décision ", Revue d'Économie Régionale et Urbaine, n² 2, p. 293-311.

Point, P. (1998), "La place de l'évaluation des biens environnementaux dans la décision publique », Économie publique, $\mathrm{n}^{\circ} 1$, p. 13-45.

Portney, P.R. (1994), "The Contingent Valuation Debate : Why Economists Should Care », Journal of Economic Perspectives, ${ }^{\circ}$ 8, p. 3-17.

Pottier, A. (2012), La forêt des Landes de Gascogne comme patrimoine naturel ? Échelles, enjeux, valeurs, Thèse de doctorat en Géographie, Université de Pau et des Pays de l'Adour, 487 p.

Rosen, S. (1974), " Hedonic prices and implicit markets : product differentiation in pure competition », Journal of Political Economy, n 82, p. 34-55.

Roy, B., Damart, S. (2002), "L'analyse Coûts-Avantages, outil de concertation et de légitimation ? ", Metropolis, $\mathrm{n}^{\circ} 108 / 109$, p. 7-16.

Rulleau, B., Rey-Valette, H. (2013), "Valuing the benefits of beach protection measures in the face of climate change : a French case-study », Journal of Environmental Economics and Policy, $\mathrm{n}^{\circ} 2$, p. 133-147.

Spash, C. (2007), «Deliberative monetary valuation (DMV) : Issues in combining economic and political processes to value environmental change », Ecological Economics, n 63, p. 690-699.

Treich, N. (2008), "L'analyse coût-bénéfice en 10 questions », Les Cahiers de la Sécurité Industrielle, $\mathrm{n}^{\circ} 3$, Toulouse, Institut pour une Culture de Sécurité Industrielle, 19 p.

Veschambre, V. (2007), "Patrimoine : un objet révélateur des évolutions de la géographie et de sa place dans les sciences sociales », Annales de géographie, n²656, p. 361-381.

Vivien F.-D. (2009), "Pour une économie patrimoniale des ressources naturelles et de l'environnement ", Monde en développement, $\mathrm{n}^{\circ}$ 145, p. 17-28.

WWF (2011), 40 ans de protection des zones humides, Paris, WWF, 40 p. 